\title{
Data Driven Scene Classification to Analyze ADAS
}

\author{
Priyadarshini R, Prof. S Kayalvizhi, A Geetha, Premkumar T
}

\begin{abstract}
In every sphere of life Big Data will be transformative. Data Visualization and Analytics plays an important role in decision making in various sectors. In autonomous vehicles data from various sensors and RADAR are stored in data logger, which is huge in size. To evaluate the performance of specific sensor manually is tedious task. This paper proposes an idea to create an interactive GUI framework to analyze the vehicle data and sensor data using big data visualization method. The framework contains various plots and plots are made interactive to analyze data in depth for all the scenarios of ADAS. It can be used to analyze the behavior of the vehicle at each instance of time interactively and time synchronized image frames are also incorporated with framework to see behavior of the plots. The paper proposes a Framework to analyze the huge amount vehicle data and sensor data which can be used to analyze the behavior of ADAS application.
\end{abstract}

Index Terms: Data Visualization, ADAS, Framework, CAN, Dash python

\section{INTRODUCTION}

Now a days, most of the applications and services use analytics tools to visualize the data. In an automobile industry vehicles are capable of producing and collecting large amount of raw data from the sensors attached to it. In industry to improve the driving experience and build smart vehicle the data science is used to analyze performance of the vehicle [1]. For autonomous driving system it is important to analyze Advance Driver Assistance System (ADAS). ADAS is the next generation electronic safety system for vehicles and autonomous cars/vehicles. Implementation of such systems will enhance the safety of the driver and co-passengers inside the vehicle and the people outside on road also. The raw data are analyzed to evaluate the performance of the vehicle [2]. There are different dashboard framework are integrated together to analyze the data from different analytics sources such as Google Analytics, Flurry, JSON and Excel files. The dashboard is created using the JSON file manually [3]. Data visualization is the presentation of data in a pictorial or graphical format. It enables the decision maker to identify difficult patterns easily [4]. Using charts or graphs it is easy to visualize large amount of complex data over spreadsheets or reports. Dash is a productive python framework to build web applications. Dash is ideal for building data visualization apps with user interface on top of Plotly.js, React.js and Flask in

Revised Manuscript Received on July 05, 2019.

Priyadarshini R, Computer Science and Engineering, Easwari Engineering College, Chennai, India.

Prof.S.Kayalvizhi, Computer Science and Engineering, Easwari Engineering College, Chennai, India.

A Geetha, Computer Science and Engineering, Easwari Engineering College, Chennai, India.

Premkumar T, System Developer Functions, ADAS AEG, WABCO Technology Center India. pure python. Dash apps are rendered in the web browser to deploy the apps in server and share them using the URL's. Dash app contains many interactive elements which makes it reactive and declarative to build complex apps easily [5].

Data visualization is another form of visual art that grabs our interest and keeps our eyes on the message. Data visualization helps to tell stories by curating data into a form easier to understand, highlighting the trends and outliers. A good visualization tells a story, removing the noise from data and highlighting the useful information. Skill sets are changing to accommodate a data-driven world. It is increasingly valuable for professionals to make decisions using data and use visuals to tell stories. With the public data visualization tools it is easy to create our own framework. There are different types of visualization graphs are available in open libraries such as Bar chart, Bubble chart, Scatter plot, word cloud etc. These are the integral part of visualizing data and a common baseline for many data graphics [6].

\section{RELATED WORK}

The paper proposes a method called VIM which is comprehensive tool for data visualization, data preprocessing and mining with drift analysis of data using web. The VIM was developed using the python Django framework and GraphLab library. VIM is built on three tier architecture such as presentation layer, Knowledge mining layer and data access layer. The dataset in CSV format is uploaded using the uploading interface of the visualization framework, when particular field is selected on clicking the button the plots are generated using GraphLab libraries for the Drift analysis. It is knowledge based system serves the analysis in comprehensive and handy way [7].

The author proposed a method to analyze the Walmart sales data, which is used to determine the business drivers and predict the different scenarios such as temperature, fuel price and holidays for which department is affected and their impact on sales at different locations. The python API framework and Scala is used to analyze the data strategies through visualization. The dataset of 3 years are used to find the relationship between the above mentioned scenarios visually. Using these analyses the retailer can manage the resources wisely [8].

The JunPing Wang et al. proposed a survey paper on new concepts of industrial big data analytics, challenges, methodologies and application. The paper focuses on five important methodologies 1.Highly distributed industrial data ingestion, 2.Industrial big data repository, 3.Large scale industrial data management, 4.Industrial data analytics and 5.Industrial data governance. For each of these methods the paper gives the challenges and

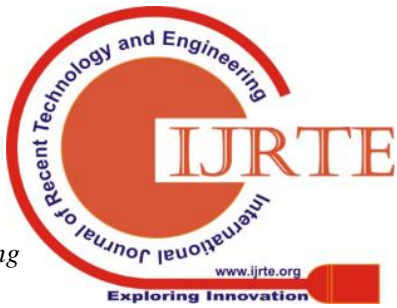


potential solutions for data analytics [9].

The paper suggest a framework in $\mathrm{R}$ tool to analyze the big data in cloud computing. $\mathrm{R}$ includes different packages and contains Deploy R server, Deploy $\mathrm{R}$ repository and Deploy $\mathrm{R}$ API's to upload and verify data. It is a flexible framework to analyze the data in cloud. At frontend java scripts are used to develop the GUI framework and the R server run tool is used to run models and display output to the user [10].

\section{Proposed Methodology}

In an Automotive vehicle RADAR and different types of sensors are used to receive the information about the driver behavior and vehicle performance. The Mileage data received are huge; hence it is difficult to analyze the performance of the system for each instance of time. This paper proposes a GUI Framework to analyze the performance of the system at different scenarios to evaluate the behavior of the Advance Driver Assistant System (ADAS). The proposed methodology consists of three major steps. First step to receive raw data from RADAR and sensors. Second step to extract and store the required signals. Third step to plot an interactive graph for required signals. The below Figure 1 describes flow of proposed methodology.

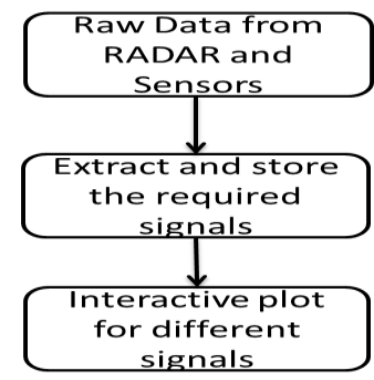

Figure 1: Flow of proposed Framework

\section{A. Raw data from RADAR and Sensors}

In vehicle the data can be collected in different ways. The Data logger is an electronic device used to record the data over time using the built in sensors available in vehicle. The logged data can be stored in any storage device from local hard drive to remote cloud storage (IOT) [11]. The data loggers are used to measure air temperature, relative humidity, AC/DC current voltage, light intensity, water temperature etc. The existed data logger in automotive industry will record CAN data from 1 channel to 16 channels, CAN FD, LIN, FlexRay, Video data, GPS data etc. The data from data loggers can be used for a. Sensor data validation, b. System algorithm validation/Resimulation, c. Driver behavior monitoring, d. Environmental parameters monitoring, e. Event justification and f. System performance validation. Control Area Network (CAN) protocol is used widely to communicate between the devices. In vehicle the CAN bus is used to send and receive the data using the CAN protocol and store the information in data logger.

\section{B. Extract and store the required signals}

The raw data collected in Data Logger from the RADAR and sensors are in unreadable format. First the data need to be converted using the CANalyzer software tool [12].
CANalyzer is intuitive and graphic block diagram depicts the data flow from the bus. In CANalyzer the measurement setup is used for the analysis of the logged data. These logged data can be stored as files for the future use. The data collected from the data logger is difficult to analyze manually, because it contains various signals from several sensors. Thus selected signals can be extracted from the logged files. Using the Graphics window of CANalyzer the required signals can be extracted based on ID of each signals. The algorithm was developed to convert the signal and store the values in files. Using these values an interactive plot is generated for each stored signals.

\section{Interactive plot for different signals}

There are several ways to design our own framework in any language. The survey papers above explain the various types of data visualization tools available for the analysis of data. The paper uses the dash libraries to create a plot interactively and the script is written in python to develop the framework. The framework contains several graphs to analyze the vehicle data.

\section{DATA VISUALIZATION FRAMEWORK}

The interactive GUI framework developed contains several main graphs for the RADAR and Sensor signals. Several dropdown buttons are created to list fields, signals, files. The graph is made to be interactive to view the particular details about the signal. On clicking the plot automatic list files are displayed. The file for which detailed analysis to be performed can be selected by using the dropdown and the subplots drawn for the signals on selected by the user. There are many options available in dash library to view and analyze the plots. In this framework Hover option is used to analyze the plot and display the required information using the json package. To analyze the behavior of the plots why such event occurred at that scenario, the image data are integrated with the plots to enhance the visualization. The algorithm is developed using Opencv library in python to integrate the image files and RADAR signal.

From the data logger, GPS data such as latitude and longitude signals are extracted and algorithm is developed to get the details of the GPS data such as weather, road type etc. using open weather map and mapquest API request method. Using these data a script is written in dash python to plot the graph. The below Figure 2 is the overview of the Framework.

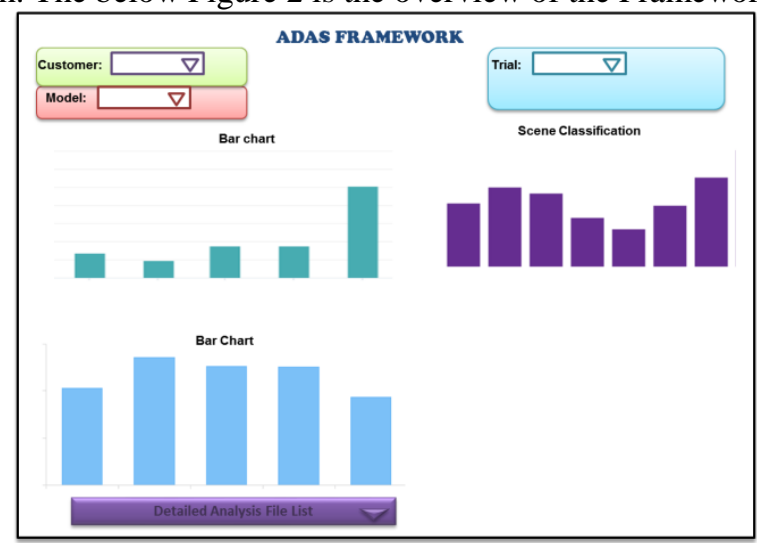

Figure 2: Overview of the Framework 


\section{CONCLUSION AND FUTURE WORK}

In an automotive industry, for autonomous vehicle it is important to analyze the performance of system behavior at each instance. In this paper an interactive framework is created using dash python script to analyze the driver behavior and vehicle behavior. To the framework, driven data are given as input and visualize the scenes for each scenario. The proposed framework analysis is done in offline mode for the driven data and as future work it can be done in online mode.

\section{ACKNOWLEDGMENT}

This project is supported and done under the guidance of ADAS team at WABCO Technology Center, Chennai, India.

\section{REFERENCES}

1. https://www.altran.com/no/en/integrated_solution/vueforge

2. https://dash.plot.ly/introduction

3. Kumar, Kapil, Joy Bose, and Sandeep Kumar Soni. "A Generic Visualization Framework based on a Data Driven Approach for the Analytics data." In 2017 14th IEEE India Council International Conference (INDICON), pp. 1-6. IEEE, 2017.

4. https://www.sas.com/en_in/insights/big-data/data-visualization.ht $\mathrm{ml}$

5. Arafat, Sk Shariful Islam, Md Shakil Hossain, Md Mahmudul Hasan, SM Al-Hossain Imam, Md Mofijul Islam, Sanjay Saha, Swakkhar Shatabda, and Tamanna Islam Juthi. "VIM: A Big Data Analytics Tool for Data Visualization and Knowledge Mining." In 2017 IEEE International WIE Conference on Electrical and Computer Engineering (WIECON-ECE), pp. 224-227. IEEE, 2017.

6. https://www.tableau.com/learn/articles/data-visualization

7. Arafat, Sk Shariful Islam, Md Shakil Hossain, Md Mahmudul Hasan, SM Al-Hossain Imam, Md Mofijul Islam, Sanjay Saha, Swakkhar Shatabda, and Tamanna Islam Juthi. "VIM: A Big Data Analytics Tool for Data Visualization and Knowledge Mining." In 2017 IEEE International WIE Conference on Electrical and Computer Engineering (WIECON-ECE), pp. 224-227. IEEE, 2017.

8. Singh, Manpreet, Bhawick Ghutla, Reuben Lilo Jnr, Aesaan FS Mohammed, and Mahmood A. Rashid. "Walmart's Sales Data Analysis-A Big Data Analytics Perspective." In 2017 4th Asia-Pacific World Congress on Computer Science and Engineering (APWC on CSE), pp. 114-119. IEEE, 2017.

9. Wang, JunPing, WenSheng Zhang, YouKang Shi, ShiHui Duan, and Jin Liu. "Industrial Big Data Analytics: Challenges, Methodologies, and Applications." arXiv preprint arXiv:1807.01016 (2018).

10. Malviya, Ayushi, Amit Udhani, and Suryakant Soni. "R-tool: Data analytic framework for big data." In 2016 Symposium on Colossal Data Analysis and Networking (CDAN), pp. 1-5. IEEE, 2016.

11. Keyur K. Patel, Sunil Patel. "IOT Based Data Logger for Monitoring and Controlling Equipment Working Status and Environmental Conditions." International Journal of Innovative Research in Computer and Communication Engineering, Vol. 4, Issue 4, April 2016.

12. https://assets.vector.com/cms/content/products/canalyzer/canaly zer/Docs/Product\%20Informations/CANalyzer_ProductInformat ion_EN.pdf 